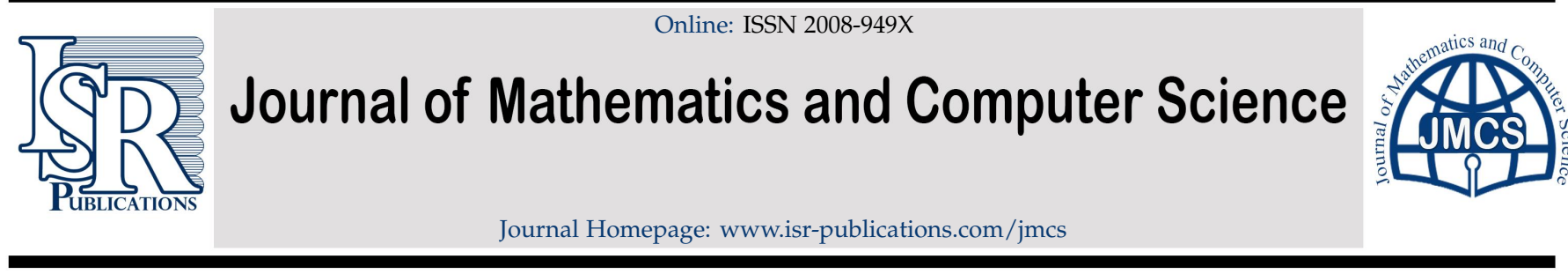

\title{
Ideal convergent sequences of functions in probabilistic normed spaces
}

\author{
Vakeel A. Khan ${ }^{a, *}$, Henna Altaf ${ }^{\mathrm{b}}$, Masood Alam ${ }^{\mathrm{c}}$ \\ ${ }^{a}$ Department of Mathematics, Aligarh Muslim University, Aligarh-202002, India. \\ ${ }^{b}$ PG Department of Mathematics, Government Degree College, Baramulla-193101, India. \\ ${ }^{c}$ Department of Mathematics and IT Center for Preparatory Studies, Sultan Qaboos University, P.O. Box 162-PC, 123 Al Khoud, Muscat, \\ Sultanate of Oman.
}

\begin{abstract}
In the present article, we have defined the notion of I-pointwise convergence and I-uniform convergence of sequence of functions defined on a probabilistic normed space with respect to the probabilistic norm $v$. Further we have given the Cauchy criteria for I-pointwise and I-uniform convergence in PNS. Also, we have proved certain results on continuity of functions with respect to $v$ in PNS.
\end{abstract}

Keywords: Ideal, filter, probabilistic normed spaces, sequence of functions.

2020 MSC: 40A35, 46S50.

(C)2022 All rights reserved.

\section{Introduction}

The notion of probabilistic normed space (PNS) is a generalisation of normed linear space in which the norm of the vectors are probability distribution functions. It was first introduced by Šerstnev in his paper [21]. Later in 1993, Alsina et al. [1] gave more generalised definition of probabilistic normed space which naturally led to definition of principal class of PN spaces, Menger spaces. The theory of PNS spaces has wide range of applications in real world problems. It has useful applications in multiple fields such as continuity properties [2], topological spaces [7], study of boundedness [16], linear operators [8], convergence of random variables [17]. The notion of statistical convergence in PN spaces was studied by Karakus [10]. Further it was extended to more generalised spaces by Esi and Savas [3-6, 20].

We insist the readers to recall the basic definitions like ideal, PNS, function sequence via references $[9,11-15,18,19]$.

\section{Main results}

In the following section, we study two types of I-convergence for sequence of functions, I-pointwise convergence and I-uniform convergence in PNS. We discuss the ideal Cauchy convergence condition for pointwise and uniform convergence.

\footnotetext{
${ }^{*}$ Corresponding author

Email address: vakhanmaths@gmail .com (Vakeel A. Khan)

doi: $10.22436 /$ jmcs.026.03.04
}

Received: 2021-09-13 Revised: 2021-09-27 Accepted: 2021-10-07 
2.1. I-pointwise convergence in PNS

Definition 2.1. Let $\left(f_{k}\right):(X, v, *) \rightarrow(Y, \mu, *)$ be a sequence of functions. $\left(f_{k}\right)$ is said to be convergent to $f$ with respect to norm $v$ if for every $t>0, \epsilon \in(0,1)$ there exists $k_{0}>0$ such that $\mu_{f_{k}(x)-f(x)}(t)>1-\epsilon$ for each $k \geqslant k_{0}$ and for each $x \in X$. We write $f_{k} \rightarrow_{v} f$.

Definition 2.2. Let $\left(f_{k}\right):(X, v, *) \rightarrow(Y, \mu, *)$ be a sequence of functions on $X$. Then $\left(f_{k}\right)$ is said to be I-pointwise convergent to a function $f$ with respect to $v$ norm if for every $x \in X, t>0$ and $\epsilon \in(0,1)$ the set

$$
\left\{k \in \mathbb{N}: \mu_{f_{k}(x)-f(x)}(t) \leqslant 1-\epsilon\right\} \in I .
$$

In this case we write $f_{k} \rightarrow_{I(v)} f$.

Theorem 2.3. Let I be an admissible ideal and $(\mathrm{X}, v, *),(\mathrm{Y}, \mu, *)$ be PN spaces. Consider a sequence of functions $\left(f_{k}\right):(X, v, *) \rightarrow(Y, \mu, *)$ such that $\left(f_{k}\right)_{k \in \mathbb{N}}$ is pointwise convergent with respect to norm $v$, then $f_{k} \rightarrow{ }_{I}(v) f$. However the converse neeed not be true.

Proof. Let $t>0$ and $\epsilon \in(0,1)$. $\left(f_{k}\right)$ is pointwise convergent to $f$ with respect to norm $v$ then there exists $k_{0}>0$ such that $\mu_{f_{k}(x)-f(x)}(t)>1-\epsilon$ for $k \geqslant k_{0}$ and for each $x \in X$.

$$
A(t, \epsilon)=\left\{k \in \mathbb{N}: \mu_{f_{k}(x)-f(x)}(t) \leqslant 1-\epsilon\right\} \subseteq\left\{1,2,3 \cdots k_{0}-1\right\} .
$$

Since $\left\{1,2,3 \cdots, k_{0}-1\right\} \in I$, we have $A(t, \epsilon) \in I$. Thus $f_{k} \rightarrow_{I(v)} f$.

The converse does not hold is proved by the following example.

Example 2.1. Let $(X,\|\|$.$) be a real normed linear space with a * b=a b$ for all $a, b \in[0,1]$ with $\mu(x, t)=$ $\frac{t}{t+\|x\|}$. Define a sequence of functions $f_{k}:[0,1] \rightarrow \mathbb{R}$ as

$$
f_{k}(x)= \begin{cases}x^{k^{2}}+1 & \text { if } k=m^{2} \text { and } x \in\left[0, \frac{1}{2}\right), \\ 0 & \text { if } k \neq m^{2} \text { and } x \in\left[0, \frac{1}{2}\right), \\ 0 & \text { if } k=m^{2} \text { and } x \in\left[\frac{1}{2}, 1\right) \\ x^{k}+\frac{1}{2} & \text { if } k \neq m^{2} \text { and } x \in\left[\frac{1}{2}, 1\right) \\ 2 & \text { if } x=1 .\end{cases}
$$

Let $A_{n}(t, \epsilon)=\left\{k \leqslant n: \mu_{f_{k}(x)-f(x)}(t) \leqslant 1-\epsilon\right\}$. Then

$$
\begin{aligned}
\mu_{f_{k}(x)-f(x)}(t) \leqslant 1-\epsilon & \Rightarrow \frac{t}{t+\left\|f_{k}(x)-f(x)\right\|} \leqslant 1-\epsilon \\
& \Rightarrow\left\|f_{k}(x)-f(x)\right\| \geqslant \frac{t \epsilon}{1-\epsilon}>0, \\
A_{n}(t, \epsilon) & =\left\{k \leqslant n:\left\|f_{k}(x)-f(x)\right\|>0\right\} \\
& =\left\{k \leqslant n: f_{k}(x)-f(x)=x^{k^{2}}+1\right\} \\
& =\left\{k \leqslant n: k=m^{2}\right\},
\end{aligned}
$$

which implies $A_{n}(t, \epsilon) \in I$. Hence for each $x \in\left[0, \frac{1}{2}\right),\left(f_{k}\right)_{k \in \mathbb{N}}$ is I-convergent to 0 with respect to probabilistic norm $v$. Similarly we can show $\left(f_{k}\right)_{k \in \mathbb{N}}$ is I-convergent to $\frac{1}{2}$ and 2 . But $\left(f_{k}\right)$ is not convergent with respect to probabilistic norm $v$.

Theorem 2.4. Let $\left(\mathrm{f}_{\mathrm{k}}\right)$ and $\left(\mathrm{g}_{\mathrm{k}}\right)$ be two function sequences from $(\mathrm{X}, \mathrm{v}, *)$ to $(\mathrm{Y}, \mu, *)$. If $\mathrm{f}_{\mathrm{k}} \rightarrow_{\mathrm{I}(v)} \mathrm{f}$ and $\mathrm{g}_{\mathrm{k}} \rightarrow_{\mathrm{I}(v)}$ $g$, then $\left(\alpha f_{k}+\beta g_{k}\right) \rightarrow_{I(v)}(\alpha f+\beta g), \alpha, \beta \in \mathbb{R}$. 
Proof. Choose $t>0$ and $\epsilon \in(0,1)$. Since $f_{k} \rightarrow_{I(v)} f$ and $g_{k} \rightarrow_{I(v)} g$ for each $x \in X$, then the sets

$$
A=\left\{k \in \mathbb{N}: \mu_{f_{k}(x)-f(x)}\left(\frac{t}{2}\right) \leqslant 1-\epsilon\right\} \in I,
$$

and

$$
\mathrm{B}=\left\{k \in \mathbb{N}: \mu_{g_{k}(x)-g(x)}\left(\frac{t}{2}\right) \leqslant 1-\epsilon\right\} \in \mathrm{I} .
$$

Thus $A^{c} \cap B^{c} \in F(I)$. Define the set

$$
C=\left\{k \in \mathbb{N}: \mu_{\left(\alpha f_{k}(x)+\beta g_{k}(x)\right)-(\alpha f(x)+\beta g(x))}(t) \leqslant 1-\epsilon\right\} .
$$

We will show $A^{c} \cap B^{c} \in C^{c}$. Let $m \in A^{c} \cap B^{c}$ then we have

$$
\begin{aligned}
\mu_{\left(\alpha f_{k}(x)+\beta g_{k}(x)\right)-(\alpha f(x)+\beta g(x))}(t) & \geqslant \mu_{\left(\alpha f_{k}(x)-\alpha f(x)\right.}\left(\frac{t}{2}\right) * \mu_{\left(\beta g_{k}(x)-\beta g(x)\right)}\left(\frac{t}{2}\right) \\
& =\mu_{f_{k}(x)-f(x)}\left(\frac{t}{2 \alpha}\right) * \mu_{g_{k}(x)-g(x)}\left(\frac{t}{2 \beta}\right) \\
& >(1-\epsilon) *(1-\epsilon) \\
& >1-\epsilon .
\end{aligned}
$$

Hence $m \in C^{c}$ implies $A^{c} \cap B^{c} \subset C^{c}$ so that $C \subset$ I and the result follows.

Definition 2.5. Let $f_{k}:(X, v, *) \rightarrow(Y, \mu, *)$ be a sequence of functions, then $\left(f_{k}\right)_{k \in \mathbb{N}}$ is called I-pointwise Cauchy sequence in PNS space if for every $t>0, \epsilon \in(0,1)$ there exists $M=M(t, \epsilon, x) \in \mathbb{N}$ such that

$$
\left\{k \in \mathbb{N}: \mu_{f_{k}(x)-f_{M}(x)}(t) \leqslant 1-\epsilon\right\} \in I .
$$

Theorem 2.6. Let $(\mathrm{X}, v, *),(\mathrm{Y}, \mu, *)$ be PN spaces and $\left(\mathrm{f}_{\mathrm{k}}\right):(\mathrm{X}, \nu, *) \rightarrow(\mathrm{Y}, \mu, *)$ be a sequence of functions. If $\left(f_{k}\right)_{k \in \mathbb{N}}$ is I-pointwise convergent sequence with respect to probabilistic norm $v$, then $\left(f_{k}\right)_{k \in \mathbb{N}}$ is I-pointwise Cauchy with respect to $v$.

Proof. Given that $\left(f_{k}\right)_{k \in \mathbb{N}}$ is I-pointwise convergent to $f$ with respect to probabilistic norm $v$ then by definition for every $t>0$ and $\epsilon \in(0,1)$ we have

$$
A=\left\{k \in \mathbb{N}: \mu_{f_{k}(x)-f(x)}\left(\frac{t}{2}\right) \leqslant 1-\epsilon\right\} \in I,
$$

which implies $A^{c} \in F(I)$. Let $k, m \in A^{c}$

$$
\begin{aligned}
\mu_{f_{k}(x)-f_{m}(x)}(t) & \geqslant \mu_{f_{k}(x)-f(x)}\left(\frac{t}{2}\right) * \mu_{f_{m}(x)-f(x)}\left(\frac{t}{2}\right) \\
& >(1-\epsilon) *(1-\epsilon) \\
& >1-\epsilon
\end{aligned}
$$

so that $\left\{k \in \mathbb{N}: \mu_{f_{k}(x)-f_{m}(x)}(t) \leqslant 1-\epsilon\right\} \in I$. Thus $\left(f_{k}\right)_{k \in \mathbb{N}}$ is I-pointwise Cauchy.

Theorem 2.7. Let $(\mathrm{X}, \mu, *),(\mathrm{Y}, \mathrm{v}, *)$ be PNS. Suppose that $\left(\mathrm{f}_{\mathrm{k}}\right):(\mathrm{X}, \mu, *) \rightarrow(\mathrm{Y}, \nu, *)$ are equi-continuous and $\left(f_{k}\right)$ is I-pointwise convergent to $f$. Then $f$ is continuous with respect to $v$.

Proof. Let $x_{0} \in X$ be fixed such that $v_{x-x_{0}}(t)>1-\epsilon$. By equi-continuity of $\left(f_{k}\right)_{k \in \mathbb{N}}$ for every $\epsilon>0$ there exists $\lambda \in(0,1)$ with $\lambda<\epsilon$ such that $\mu_{f_{k}(x)-f_{k}\left(x_{0}\right)}\left(\frac{t}{3}\right)>1-\lambda$ for every $k \in \mathbb{N}$. Since $f_{k} \rightarrow_{I(v)} f$ we have

$$
\left\{k \in \mathbb{N}: \mu_{f_{k}\left(x_{0}\right)-f\left(x_{0}\right)}\left(\frac{t}{3}\right) \leqslant 1-\lambda\right\} \cup\left\{k \in \mathbb{N}: \mu_{f_{k}(x)-f(x)}\left(\frac{t}{3}\right) \leqslant 1-\lambda\right\} \in I .
$$


We have,

$$
\begin{aligned}
\mu_{f\left(x_{0}\right)-f(x)}(t) & \geqslant \mu_{f\left(x_{0}\right)-f_{k}\left(x_{0}\right)}\left(\frac{t}{3}\right) * \mu_{f_{k}\left(x_{0}\right)-f_{k}(x)}\left(\frac{t}{3}\right) * \mu_{f_{k}(x)-f(x)}\left(\frac{t}{3}\right) \\
& >(1-\lambda) *(1-\lambda) *(1-\lambda) \\
& >(1-\lambda) *(1-\lambda) \\
& >(1-\lambda) \\
& >1-\epsilon
\end{aligned}
$$

and the continuity of $f$ is proved.

\subsection{I-uniform convergence in PNS}

Definition 2.8. Let $I \subset P(\mathbb{N})$ be an admissible ideal and $\left(f_{k}\right):(X, v, *) \rightarrow(Y, \mu, *)$ is said to be I-uniform convergent to $f$ with respect to probabilistic norm $v$ if and only if for all $t>0$ there exists $M \subset \mathbb{N}, M \in F(I)$ and $k_{0}=k_{0}(t, \epsilon, x) \in M$, for all $k>k_{0}$ and $k \in M$ and for all $x \in X, \epsilon \in(0,1)$,

$$
\left\{k \leqslant n: \mu_{f_{k}(x)-f(x)}(t) \leqslant 1-\epsilon\right\} \in I .
$$

We write $f_{k} \rightrightarrows I(v)$ f.

Definition 2.9. Let $(X, v, *),(Y, \mu, *)$ be probabilistic normed spaces and $\left(f_{k}\right):(X, v, *) \rightarrow(Y, \mu, *), f$ : $(X, v, *) \rightarrow(Y, \mu, *)$ be a bounded function. Then $f_{k} \rightrightarrows I(v) f$ if and only if $I-\lim \left(\inf _{x \in X} \mu_{f_{k}(x)-f(x)}(t)\right)=1$.

Remark 2.10. If $f_{k} \rightrightarrows_{I(v)} f$ then $f_{k} \rightarrow_{I(v)} f$. But the converse is not true.

Example 2.2. Define a sequence of functions

$$
f_{k}(x)= \begin{cases}0, & \text { if } k=n^{2}, \\ \frac{k^{2} x}{1+k^{3} x^{2}}, & \text { otherwise, }\end{cases}
$$

on $[0,1] . f_{k}\left(\frac{1}{k}\right) \rightarrow_{I(v)} 1$ and $f_{k}(0) \rightarrow_{I(v)} 0$. The sequence is I-pointwise convergent to 0 with respect to probabilistic norm $v$, but it is not I-uniform convergent with respect to $v$.

Theorem 2.11. Let $\mathrm{I} \subset \mathrm{P}(\mathbb{N})$ be an admissible ideal. Assume that $\left(\mathrm{f}_{\mathrm{k}}\right):(\mathrm{X}, v, *) \rightarrow(\mathrm{Y}, \mu, *)$ is uniformly convergent to $\mathrm{f}$, then $\left(\mathrm{f}_{\mathrm{k}}\right)$ is I-uniformly convergent to $\mathrm{f}$ with respect to $\mathrm{v}$.

Proof. Suppose that the sequence of functions $\left(f_{k}\right):(X, v, *) \rightarrow(Y, \mu, *)$ is uniformly convergent to $f$ : $(\mathrm{X}, v, *) \rightarrow(\mathrm{Y}, \mu, *)$ with respect to probabilstic norm $v$. Then by definition for every $\mathrm{t}>0, \epsilon \in(0,1)$ there exists a positive integer $k_{0}=k_{0}(t, \epsilon)$ such that for all $x \in X$ and $k>k_{0}, \mu_{f_{k}(x)-f(x)}(t)>1-\epsilon$, so that

$$
A(t, \epsilon)=\left\{k \in \mathbb{N}: \mu_{f_{k}(x)-f(x)}(t) \leqslant 1-\epsilon\right\} \subseteq\left\{1,2,3, \cdots, k_{0}\right\} \in I .
$$

Hence for every $t>0$, there exists $A^{c} \subset \mathbb{N}, A^{c} \in F(I)$ and there exists $k_{0}(t, \epsilon) \in A^{c}$ such that for all $k>k_{0}$ with $k \in A^{c}$ and for all $x \in X, \mu_{f_{k}(x)-f(x)}(t)>1-\epsilon$. This implies $f_{k} \rightrightarrows_{I(v)} f$.

Definition 2.12. let $(X, v, *),(Y, \mu, *)$ be PN spaces and let $\left(f_{k}\right):(X, v, *) \rightarrow(Y, \mu, *)$ be a sequence of functions. Then $\left(f_{k}\right)$ is said to be I-uniform Cauchy sequence with respect to $v$ if for every $t>0, \epsilon \in(0,1)$, there exists $N=N(t, \epsilon) \in \mathbb{N}$ such that

$$
\left\{k \in \mathbb{N}: \mu_{f_{k}(x)-f_{N}(x)}(t) \leqslant 1-\epsilon\right\} \in I .
$$

Theorem 2.13. If $\left(f_{k}\right):(X, v, *) \rightarrow(Y, \mu, *)$ be I-uniform convergent with respect to $v$, where $(X, v, *)$ and $(\mathrm{Y}, \mu, *)$ are probabilistic normed spaces, then $\left(\mathrm{f}_{\mathrm{k}}\right)$ is I-uniform Cauchy with respect to probabilistic norm $v$. 
Proof. Given that $f_{k} \rightrightarrows I(v)$ f. By definition for every $t>0, \epsilon \in(0,1)$ there exists $A \subset \mathbb{N}, A \in F(I)$ and $k_{0}(t, \epsilon) \in A$ such that for all $k>k_{0}, k \in A$ and for all $x \in X, \mu_{f_{k}(x)-f(x)}\left(\frac{t}{2}\right)>1-\epsilon$. Choose $N=N(t, \epsilon), N>k_{0}$, so that $\mu_{f_{N}(x)-f(x)}\left(\frac{t}{2}\right)>1-\epsilon$.

$$
\begin{aligned}
\mu_{f_{k}(x)-f_{N}(x)}(t) & >\mu_{f_{k}(x)-f(x)}\left(\frac{t}{2}\right) * \mu_{f(x)-f_{N}(x)}\left(\frac{t}{2}\right) \\
& >(1-\epsilon) *(1-\epsilon) \\
& >1-\epsilon .
\end{aligned}
$$

Thus $\left\{k \in \mathbb{N}: \mu_{f_{k}(x)-f_{N}(x)}(t) \leqslant 1-\epsilon\right\} \in I$. Hence $\left(f_{k}\right)$ is I-uniform Cauchy sequence in PNS.

Theorem 2.14. Let $(\mathrm{X}, v, *)$ and $(\mathrm{Y}, \mu, *)$ be PN spaces. Suppose $\left(\mathrm{f}_{\mathrm{k}}\right):(\mathrm{X}, v, *) \rightarrow(\mathrm{Y}, \mu, *), \mathrm{k} \in \mathbb{N}$ be continuous with respect to probabilistic norm $v$. If $\mathrm{f} \rightrightarrows \mathrm{I}(v) \mathrm{f}$, then $\mathrm{f}$ is continuous.

Proof. Fix $x_{0} \in X$ such that $v_{x_{0}-x}(t)>1-\epsilon$. By continuity of $f_{k}$, for every $t>0$ there exists $\lambda \in(0,1)$ with $\lambda<\epsilon$ such that $\mu_{f_{k}\left(x_{0}\right)-f_{k}(x)}\left(\frac{t}{3}\right)>1-\lambda$. Since $f_{k} \rightrightarrows I(v) f$, we have

$$
\left\{k \in \mathbb{N}: \mu_{f_{k}(x)-f(x)}\left(\frac{t}{3}\right) \leqslant 1-\lambda\right\} \cup\left\{k \in \mathbb{N}: \mu_{f_{k}\left(x_{0}\right)-f\left(x_{0}\right)}\left(\frac{t}{3}\right) \leqslant 1-\lambda\right\} \in I .
$$

So, there exists $m \in F(I)$ such that $\mu_{f_{m}(x)-f(x)}\left(\frac{t}{3}\right)>1-\lambda$ and $\mu_{f_{m}\left(x_{0}\right)-f\left(x_{0}\right)}\left(\frac{t}{3}\right)>1-\lambda$.

$$
\begin{aligned}
\mu_{\mathrm{f}(x)-f\left(x_{0}\right)}(t) & \geqslant \mu_{f(x)-f_{m}(x)}\left(\frac{t}{3}\right) * \mu_{f_{m}(x)-f_{m}\left(x_{0}\right)}\left(\frac{t}{3}\right) * \mu_{f_{m}\left(x_{0}\right)-f_{(x 0)}}\left(\frac{t}{3}\right) \\
& >(1-\lambda) *(1-\lambda) *(1-\lambda) \\
& >(1-\lambda) *(1-\lambda) \\
& >1-\lambda \\
& >1-\epsilon .
\end{aligned}
$$

Thus $f$ is continuous with respect to probabilistic norm $v$.

\section{Acknowledgement}

The authors would like to thank the referees and the editor for their careful reading and their valuable comments.

\section{References}

[1] C. Alsina, B. Schweizer, A. Sklar, On the definition of a probabilistic normed space, Aequationes Math., 46 (1993), 91-98. 1

[2] C. Alsina, B. Schweizer, A. Sklar, Continuity properties of probabilistic norms, J. Math. Anal. Appl., 208 (1997), 446452. 1

[3] A. Esi, On Asymptotically Lacunary Statistical Equivalent Sequences in Probabilistic Normed Space, J. Math. Stat., 9 (2013), 144-148. 1

[4] A. Esi, On Almost Asymptotically Lacunary Statistical Equivalent Sequences Induced Probabilistic Norms, Analysis (Berlin), 34 (2014), 19-28.

[5] A. Esi, M. K. Özdemir, Generalized Delta (m)-Statistical Convergence in Probabilistic Normed Space, J. Comput. Anal. Appl., 13 (2011), 923-932.

[6] A. Esi, M. K. Özdemir, Lacunary Statistical Convergence of Double Generalized Difference Sequences on Probabilistic Normed Space, J. Math. Comput. Sci., 2 (2012), 23-36. 1 
[7] M. J. Frank, Probabilistic topological spaces, J. Math. Anal. Appl., 34 (1971), 67-81. 1

[8] J. A. Fridy, Statistical limit points, Proc. Amer. Math. Soc., 118 (1993), 1187-1192. 1

[9] B. Hazarika, S. A. Mohiuddine, Ideal convergence of random variables, J. Funct. Spaces Appl., 2013 (2013), 7 pages. 1

[10] S. Karakus, Statistical convergence on probalistic normed spaces, Math. Commun., 12 (2007), 11-23. 1

[11] V. A. Khan, K. Ebadullah, Yasmeen, On Zweier I-convergent sequence spaces, Proyecciones, 33 (2014), 259-276. 1

[12] Ö. Kişi, Lacunary ideal convergence in measure for sequences of fuzzy valued functions, J. Intel. Fuzzy Syst., 40 (2021), 5517-5526.

[13] P. Kostyrko, T. Salát, W. Wilczyńskis, I-convergence, Real Anal. Exchange, 26 (2000/01), 669-686.

[14] B. Lafuerza Guillen, P. Harikrishnan, Probabilistic normed spaces, World Scientific, London, (2014).

[15] B. Lafuerza-Guillen, M. Rafi, Statistical convergence in strong topology of probabilistic normed spaces, Note Mat., 29 (2009), 79-88. 1

[16] B. Lafuerza Guillen, J. A. Rodriguez Lallena, C. Sempi, A study of boundedness in probabilistic normed spaces, J. Math. Anal. Appl., 232 (1999), 183-196. 1

[17] B. Lafuerza-Guillen, C. Sempi, Probabilistic norms and convergence of random variables, J. Math. Anal. Appl., 280 (2003), 9-16. 1

[18] M. Mursaleen, S. A. Mohiuddine, On ideal convergence of double sequences in probabilistic normed spaces, Math. Rep. (Bucur.), 12 (2010), 359-371. 1

[19] M. Mursaleen, S. A. Mohiuddine, On ideal convergence in probabilistic normed spaces, Math. Slovaca, 62 (2012), 49-62. 1

[20] E. Savaş, A. Esi, Statistical Convergence of Triple Sequences on Probabilistic Normed Space, An. Univ. Craiova Ser. Mat. Inform., 39 (2012), 226-236. 1

[21] A. N. Šerstnev, Random normed spaces. Questions of completeness, (Russian) Kazan. Gos. Univ. Učen. Zap., 122 (1962), 3-20. 1 